\title{
The Effects of Monomer, Crosslinking Agent, and Filler Concentrations on the Viscoelastic and Swelling Properties of Poly(methacrylic acid) Hydrogels: A Comparison
}

\author{
Claudia Mihaela Ninciuleanu ${ }^{1,2}$, Raluca Ianchiş ${ }^{1}$ (D) Elvira Alexandrescu ${ }^{1}$ (D), Cătălin Ionuţ Mihăescu ${ }^{1}$, \\ Cristina Scomoroşcenco ${ }^{1}$, Cristina Lavinia Nistor ${ }^{1}$ (D), Silviu Preda ${ }^{3} \mathbb{D}$, Cristian Petcu ${ }^{1, *(D)}$ \\ and Mircea Teodorescu $2, *$ (D)
}

Citation: Ninciuleanu, C.M.; Ianchis, R.; Alexandrescu, E.; Mihăescu, C.I.; Scomoroşcenco, C.; Nistor, C.L.; Preda, S.; Petcu, C.; Teodorescu, M. The Effects of Monomer, Crosslinking Agent, and Filler Concentrations on the Viscoelastic and Swelling Properties of Poly(methacrylic acid) Hydrogels: A Comparison. Materials 2021, 14, 2305. https://doi.org/ $10.3390 /$ ma14092305

\section{Academic Editors:}

Giuseppina Sandri, Franz E. Weber and Isabel Izquierdo-Barba

Received: 10 March 2021

Accepted: 27 April 2021

Published: 29 April 2021

Publisher's Note: MDPI stays neutral with regard to jurisdictional claims in published maps and institutional affiliations.

Copyright: (c) 2021 by the authors Licensee MDPI, Basel, Switzerland. This article is an open access article distributed under the terms and conditions of the Creative Commons Attribution (CC BY) license (https:/ / creativecommons.org/licenses/by/ $4.0 /)$.
1 National Institute for Research and Development in Chemistry and Petrochemistry-ICECHIM, Spl. Independentei 202, 060021 Bucharest, Romania; claudia.ninciuleanu@yahoo.com (C.M.N.); ralumoc@yahoo.com (R.I.); elviraalexandrescu@yahoo.com (E.A.); mihaescu_catalin96@yahoo.com (C.I.M.); scomoroscencocristina@gmail.com (C.S.); lc_nistor@yahoo.com (C.L.N.)

2 Department of Bioresources and Polymer Science, Faculty of Applied Chemistry and Materials Science, Politehnica University of Bucharest, 1-7 Gh. Polizu Street, 011061 Bucharest, Romania

3 Institute of Physical Chemistry "Ilie Murgulescu", Romanian Academy, Spl. Independentei 202, 6th District, P.O. Box 194, 060021 Bucharest, Romania; predas01@yahoo.co.uk

* Correspondence: cpetcu@icf.ro (C.P.); mircea.teodorescu@upb.ro (M.T.)

Abstract: The present work aims at comparatively studying the effects of the concentrations of a monomer (10-30 $\mathrm{wt} \%$ based on the whole hydrogel composition), crosslinking agent (1-3 mol\% based on the monomer), and reinforcing agent (montmorillonite-MMT, 1-3 wt.\% based on the whole hydrogel composition) on the swelling and viscoelastic properties of the crosslinked hydrogels prepared from methacrylic acid (MAA) and $\mathrm{N}, \mathrm{N}^{\prime}$-methylenebisacrylamide (BIS) in the presence of $\mathrm{K}_{2} \mathrm{~S}_{2} \mathrm{O}_{8}$ in aqueous solution. The viscoelastic measurements, carried out on the as-prepared hydrogels, showed that the monomer concentration had the largest impact, its three-time enhancement causing a 30-fold increase in the storage modulus, as compared with only a fivefold increase in the case of the crosslinking agent and 1.5-fold increase for MMT in response to a similar threefold concentration increase. Swelling studies, performed at three $\mathrm{pH}$ values, revealed that the water absorption of the hydrogels decreased with increasing concentration of both the monomer and crosslinking agent, with the amplitude of the effect of concentration modification being similar at $\mathrm{pH} 5.4$ and 7.4 in both cases, but very different at $\mathrm{pH}$ 1.2. Further, it was shown that the increased $\mathrm{pH}$ differently influenced the swelling degree in the case of the hydrogel series in which the concentrations of the monomer and crosslinking agent were varied. In contrast to the effect of the monomer and crosslinking agent concentrations, the increase in the MMT amount in the hydrogel resulted in an increased swelling degree at $\mathrm{pH} 5.4$ and 7.4, while at $\mathrm{pH}$ 1.2, a slight decrease in the water absorption was noticed. The hydrogel crosslinking density determinations revealed that this parameter was most affected by the increase in the monomer concentration.

Keywords: poly(methacrylic acid); composite hydrogels; viscoelastic properties; swelling degree; concentration effects

\section{Introduction}

Hydrogels can be defined as hydrophilic polymeric networks formed from natural and/or synthetic polymers that are capable of absorbing and retaining a large amount of water or aqueous fluids without dissolving due to the crosslinked structure [1]. Over time, a wide variety of hydrogels have been obtained with applications in various fields such as medicine and pharmacy (drug delivery, tissue engineering, wound healing) [2], agriculture [3], electronic equipment industry [4], cosmetics industry [5], etc. The most important properties of hydrogels that must be taken into account in any application are 
the water content and the mechanical/viscoelastic properties, to which, depending on the use, others properties may be added, such as biocompatibility in the case of medical uses. However, the mechanical properties of hydrogels are generally low, which is a major disadvantage in many situations. The simplest methods of improving the mechanical properties of common hydrogels are based on adjusting the crosslinking degree by either changing the concentration of the monomer introduced in the synthesis process or by altering the crosslinking agent-monomer ratio, or adding a reinforcing agent; the water absorption of the hydrogels is simultaneously affected in all these cases [2,6,7].

Poly(methacrylic acid) (PMAA) hydrogels are $\mathrm{pH}$-sensitive anionic materials with mucoadhesive properties. Because of this, they have found many applications in the drug-controlled release field, as they ensure protection to the drugs inside the stomach at acidic $\mathrm{pH}[8,9]$. Garcia et al. [8] studied PMAA hydrogels for the controlled release of metoclopramide by varying the concentration of $\mathrm{N}, \mathrm{N}^{\prime}$-methylenebisacrylamide (BIS) as a crosslinking agent. Following this study, the authors noted that the swelling degree decreased with an increase in the concentration of the crosslinking agent. A more detailed study of PMAA hydrogels was performed by Panis et al. [7], who showed that the xerogel density, the average molar mass between the crosslinking points, and the distance between the macromolecular chains increased, while the crosslinking degree decreased with increasing neutralization degree of the monomer. The methacrylic acid (MAA) concentration had a similar effect on the swelling degree, i.e., when a higher concentration of the monomer was used, the swelling degree decreased because more crosslinking points were formed in the network, leading to a matrix with a higher crosslinking density [7].

The literature also describes hydrogels based on PMAA that have been studied in combination with natural polymers (chitosan [10], starch [11], Salecan [12]), as well as copolymers of MAA with different monomers (acrylic acid [13], N-isopropylacrylamide [14], methacrylamide [15]) or hydrogels based on PMAA and poly(ethylene glycol) [16]. In the majority of cases, the hydrogels obtained were tested for the controlled release of various drugs such as theophylline, doxorubicin, and insulin. For example, Bajpai and Singh [15] investigated the swelling behavior of some poly (methacrylamide-co-MAA) hydrogels synthesized under various conditions and showed that by increasing the crosslinking agent amount, the swelling degree decreased. They also investigated the swelling behavior at different $\mathrm{pH}$ values (1-8) and noticed that the highest swelling degree was obtained for a $\mathrm{pH}$ value equal to 8 , but a major change in volume of the hydrogels occurred starting with the 6-7 $\mathrm{pH}$ interval. Another important conclusion referred to the initial polymerization medium: when it was more diluted, the swelling degree increased because loop formation was favored against the creation of crosslinking points in the hydrogel network.

Mechanical properties are very important in the case of hydrogels used for controlled release, as well as for any other field. These properties can be improved by adding reinforcing agents such as clays (montmorillonite, laponite, kaolin etc.). Montmorillonite (MMT) is a natural clay that belongs to the smectite category, displaying the empirical formula $\mathrm{Al}_{2} \mathrm{Si}_{4} \mathrm{O}_{10}(\mathrm{OH})_{2} \times \mathrm{yH}_{2} \mathrm{O}$. Its employment for the reinforcement of hydrogels is more advantageous economically because MMT is both readily available in nature and is environmentally friendly. Another important advantage of MMT is that it has a hydrophilic character and can be easily dispersed in the presence of hydrophilic polymers. Zhumagaliyeva et al. obtained bentonite-reinforced poly(acrylic acid)/PMAA composite hydrogels and studied the influence of several factors (temperature, crosslinking degree, clay content) on the swelling properties of hydrogels. They concluded that the swelling degree increased with $\mathrm{pH}$ and temperature and decreased with the concentration of the reinforcing agent [17]. Further, Junior et al. synthesized and characterized intercalated nanocomposite hydrogels based on PMAA and different concentrations of MMT (Cloisite $\mathrm{Na}, 5-20 \mathrm{wt} . \%$ ) with potential applications in agriculture as controlled release systems [18] and studied the effect of clay concentration and environmental salinity on the swelling properties. Ianchis et al. studied PMAA composite hydrogels reinforced with commercial clay C193A [19], as well as the effect of several types of commercial clays, namely, 
$\mathrm{ClNa}$ (unmodified montmorillonite) and various organomodified types of MMT (Cl30B, $\mathrm{Cl} 20 \mathrm{~A}$, and $\mathrm{Cl15A}$ ) on the hydrogel network made up of PMAA and Salecan [20]. It was observed that $\mathrm{ClNa}$ was the most compatible clay with this type of network due to its hydrophilic character.

Crosslinked hydrogels based on PMAA or PMAA and MMT have potential uses in the controlled release of drugs [8,10-12] or agrochemicals [18] and wastewater purification [9].

The present work aimed to compare the effects of the monomer (MAA) and reinforcing agent (MMT) concentrations and crosslinking agent (BIS)/monomer (MAA) ratio on the viscoelastic properties and water absorption at various $\mathrm{pH}$ values of the crosslinked PMAA hydrogels. To the best of our knowledge, this is the first report of such a study. For a better comparison of the effect of the various components, the viscoelastic properties of hydrogels were investigated immediately after synthesis ("as-prepared hydrogels"), when their composition was identical to that of the precursor solution, while the water absorption/swelling degree was calculated in relation to the amount of polymer in the hydrogel only. We will show within this paper that, out of the three components mentioned which were employed within the usual concentration limits, the monomer concentration used in the synthesis process displhadayed the most pronounced influence on both the viscoelastic properties and crosslinking density of hydrogels. Further, the swelling degree of the PMAA hydrogels decreased with the concentration of the monomer and crosslinking agent and increased with the concentration of MMT; the resulting values depended on the $\mathrm{pH}$ of the medium, in agreement with the $\mathrm{pH}$-sensitive nature of these types of hydrogels.

\section{Materials and Methods}

\subsection{Materials}

Unmodified montmorillonite (Cloisite $\mathrm{Na}, \mathrm{ClNa}$ ) provided by Southern Clay Products Inc. (Gonzales, TX, USA) was purified by magnetic stirring with distilled water $(100 \mathrm{~g} / 1 \mathrm{~L}$ distilled water) at room temperature for $24 \mathrm{~h}$, followed by decantation for $24 \mathrm{~h}$. The sedimented part was separated, and the supernatant was centrifuged at $900 \mathrm{rpm}$ for $30 \mathrm{~min}$; the collected sediment was dried by lyophilization and used in experiments. Methacrylic acid (MAA, 99\% Janssen Chimica, Beerse, Belgium), N, N methylenebisacrylamide (BIS 99\% Sigma Aldrich, Saint Louis, MS, USA), and ammonium persulfate (APS 98\% Sigma Aldrich) were used as received. The solvent used in the synthesis of hydrogels was deionized water (DI, 18.2 M $\Omega$ resistivity). Swelling studies were performed in deionized water ( $\mathrm{pH}$ 5.4), $\mathrm{HCl}$ solution ( $\mathrm{pH}$ 1.2), and PBS (phosphate-buffered saline) solution (0.01 M, pH 7.4) obtained from tablets (Sigma) and deionized water according to the manufacturer's instructions and adjusted as needed to obtain the desired $\mathrm{pH}$.

\subsection{Synthesis of Hydrogels}

The clay was dispersed under magnetic stirring in the calculated amount of DI for $24 \mathrm{~h}$, and the monomer and crosslinking agent were added, followed by the initiator. The polymerization mixture was then injected into a mold consisting of two glass plates separated by a 1-mm-thick Teflon gasket. The mold was sealed and placed in a thermostatted bath at $60^{\circ} \mathrm{C}$ for $5 \mathrm{~h}$. At the end of the polymerization process, the mold was cooled down, disassembled, and discs of $8 \mathrm{~mm}$ and $20 \mathrm{~mm}$ in diameter were cut from the hydrogel. The $8 \mathrm{~mm}$ discs were purified for seven days in DI, with the water being replaced every day, while the $20 \mathrm{~mm}$ discs were used immediately for rheological measurements. Table 1 displays the compositions of the investigated hydrogels. Three different series of hydrogels were investigated: series 1: variation in the monomer concentration (10, 20, and $30 \mathrm{wt} \%$ MAA based on the whole hydrogel composition); series 2: variation of the concentration of the crosslinking agent $(1,2,3 \mathrm{~mol} \%$ based on the monomer); series 3 : variation of the filler (MMT) concentration (1, 2, $3 \mathrm{wt} \%$ based on the whole hydrogel composition). 
Table 1. The composition of the hydrogels investigated.

\begin{tabular}{|c|c|c|c|c|c|c|c|c|c|}
\hline $\begin{array}{c}\text { Sample } \\
\text { Code }\end{array}$ & $\begin{array}{l}\mathrm{H}_{2} \mathrm{O} \\
(\mathrm{g})\end{array}$ & $\begin{array}{c}\text { MAA } \\
\text { (g) }{ }^{1}\end{array}$ & $\underset{(w t \%)^{2}}{\text { MAA }}$ & $\begin{array}{l}\text { BIS } \\
(g)^{1}\end{array}$ & $\begin{array}{c}\text { BIS } \\
(\operatorname{mole} \%)^{3}\end{array}$ & $\begin{array}{l}\text { APS } \\
\text { (g) }{ }^{1}\end{array}$ & $\begin{array}{c}\text { APS } \\
(\mathrm{mole} \%)^{3}\end{array}$ & $\begin{array}{l}\text { MMT } \\
(\mathrm{g})^{1}\end{array}$ & $\begin{array}{l}\text { MMT } \\
w t \% 4\end{array}$ \\
\hline$S H^{5}$ & 89.4 & 10 & 10 & 0.36 & 2 & 0.26 & 1 & 0 & 0 \\
\hline H $20 \%$ MAA & 78.8 & 20 & 20 & 0.72 & 2 & 0.52 & 1 & 0 & 0 \\
\hline H30\%MAA & 48.16 & 30 & 30 & 1.08 & 2 & 0.76 & 1 & 0 & 0 \\
\hline H1\%BIS & 89.6 & 10 & 10 & 0.18 & 1 & 0.26 & 1 & 0 & 0 \\
\hline H3\%BIS & 89.2 & 10 & 10 & 0.54 & 3 & 0.26 & 1 & 0 & 0 \\
\hline H1\%MMT & 88.4 & 10 & 10 & 0.36 & 2 & 0.26 & 1 & 1 & 9.65 \\
\hline $\mathrm{H} 2 \% \mathrm{MMT}$ & 87.4 & 10 & 10 & 0.36 & 2 & 0.26 & 1 & 2 & 19.3 \\
\hline H3\%MMT & 86.4 & 10 & 10 & 0.36 & 2 & 0.26 & 1 & 3 & 28.95 \\
\hline
\end{tabular}

${ }^{1}$ For $100 \mathrm{~g}$ amount reaction mixture; ${ }^{2}$ Based on the whole reaction mass; ${ }^{3}$ With respect to MAA; ${ }^{4}$ With respect to the total monomer concentration; ${ }^{5}$ Standard hydrogel (the hydrogel with the standard composition: $10 \%$ MAA-2\%BIS-0\%MMT).

To determine the monomer conversion, part of the hydrogel was dried first in air and then over anhydrous $\mathrm{CaCl}_{2}$ to a constant mass and weighed (Wo). The dry xerogel was then immersed for purification in DI, which was changed daily for seven days, and then dried again (Wext). The monomer conversion (C\%) was calculated according to Equation (1):

$$
\mathrm{C}(\%)=\left(\mathrm{Wext}-\mathrm{W}_{\mathrm{MMT}}\right) /\left(\mathrm{Wo}-\mathrm{W}_{\mathrm{MMT}}\right) \times 100
$$

where $\mathrm{W}_{\mathrm{MMT}}$ is the amount of MMT contained in the material used to determine the conversion.

\subsection{Determination of Swelling Degree and Crosslinking Density}

To determine the swelling degree, the pre-weighed xerogels $\left(\mathrm{W}_{\mathrm{X}}\right)$ were immersed in $30 \mathrm{~mL}$ of swelling solution at $37^{\circ} \mathrm{C}$ for $72 \mathrm{~h}$ or other various time intervals. The hydrogels were then removed from the solution, wiped superficially with filter paper, and weighed $\left(\mathrm{W}_{\mathrm{H}}\right)$. All the measurements were performed in duplicate. The swelling degree (SD) was calculated as the ratio of the amount of water absorbed to the mass of polymer in xerogel according to Equation (2):

$$
\mathrm{SD}(\mathrm{g} / \mathrm{g})=\left(\mathrm{W}_{\mathrm{H}}-\mathrm{W}_{\mathrm{X}}\right) /\left(\mathrm{W}_{\mathrm{X}} \times(100-\% \mathrm{MMT} / 100)\right)
$$

where \% MMT is the percentage of MMT in xerogel, calculated based on the amount of monomers and MMT introduced in the polymerization and the total monomer conversion.

The average molecular weight between crosslinks $\left(\overline{\mathrm{M}}_{\mathrm{c}}\right)$ and the crosslinking density $\left(\rho_{c}\right)$ were determined in the case of the hydrogels swelled at $\mathrm{pH} 1.2$ in order to avoid the influence of the base character of the aqueous MMT dispersion on the swelling degree of the composite hydrogels (see below). At pH 1.2, all the methacrylic acid units within the hydrogel were in the acid form, and therefore the hydrogels can be considered non-ionic. In the case of non-ionic hydrogels and neglecting the contribution of the chain-end defects, $\overline{\mathrm{M}}_{\mathrm{c}}$ can be calculated according to Equation (3) [21,22].

$$
\overline{\mathrm{M}}_{\mathrm{c}}=-\frac{\left(1-\frac{2}{\mathrm{f}}\right) \mathrm{V}_{1} v_{2 \mathrm{r}}^{2 / 3} v_{2 \mathrm{~m}}^{1 / 3}}{\bar{v}\left[\ln \left(1-v_{2 \mathrm{~m}}\right)+v_{2 \mathrm{~m}}+\chi v_{2 \mathrm{~m}}^{2}\right]}
$$

where $\mathrm{f}$ is the functionality of the crosslinking site $(\mathrm{f}=4), \mathrm{V}_{1}$ represents the molar volume of the solvent (for water $V_{1}=18 \mathrm{~cm}^{3} / \mathrm{mol}$ ), $v_{2 \mathrm{r}}$ and $v_{2 \mathrm{~m}}$ represent the polymer volume fraction of the as-prepared hydrogel and the hydrogel swelled at equilibrium, respectively, $\bar{v}$ is the specific volume of the polymer $\left(0.775 \mathrm{~cm}^{3} / \mathrm{g}\right.$ in the case of PMAA), while $\chi$ represents the Flory polymer-solvent interaction factor $(0.48$ for water-PMAA at $0 \%$ degree of ionization [23].

The polymer volume fractions $\left(v_{2 r}, v_{2 m}\right)$ were calculated by using Equation (4) [24]

$$
v_{2 \mathrm{r} / \mathrm{m}}=\left[1+\frac{\rho_{\mathrm{p}}}{\rho_{\mathrm{s}}} \times\left(\frac{\mathrm{W}_{\mathrm{h}}}{\mathrm{W}_{\mathrm{x}}}-1\right)\right]^{-1}
$$


where $\rho_{\mathrm{p}}$ and $\rho_{\mathrm{s}}$ are the polymer $\left(1.29 \mathrm{~g} / \mathrm{cm}^{3}\right.$ for PMAA [25] and solvent densities $\left(1.00 \mathrm{~g} / \mathrm{cm}^{3}\right.$ in the case of water), while $W_{h}$ and $W_{x}$ represent the mass of the hydrogel at equilibrium swelling $\left(v_{2 \mathrm{~m}}\right)$ or under as-prepared conditions $\left(v_{2 \mathrm{r}}\right)$, and the mass of the corresponding xerogel, respectively.

The crosslinking density of the hydrogels was calculated according to Equation (5) [24].

$$
\rho_{\mathrm{c}}=\frac{1}{\bar{v} \times \overline{\mathrm{M}}_{\mathrm{c}}}
$$

\subsection{Characterization of the Hydrogels}

The rheological measurements were carried out at $25^{\circ} \mathrm{C}$ on a Kinexus Pro instrument (Malvern Instruments, Malvern, UK, software 1.6) by using $20 \mathrm{~mm}$ parallel plates with roughened surface to avoid slippage. The normal force applied was $0.5 \mathrm{~N}$. The amplitude sweep measurements were performed a $1 \mathrm{~Hz}$ constant frequency, while the frequency sweep experiments were carried out in the $0.1-10 \mathrm{~Hz}$ range, a deformation within the linear viscoelasticity range being applied.

The FTIR spectra were recorded on a Tensor 37 Bruker equipment (Woodstock, NY, USA) equipped with a Golden Gate ATR unit, by using ground xerogels.

X-ray diffraction (XRD) measurements were carried out on a Rigaku Ultima IV X-ray diffractometer (Tokyo, Japan) operated at $40 \mathrm{kV}$ and $30 \mathrm{~mA}$ by employing xerogel powders.

The thermogravimetric analysis (TGA) of the xerogels was performed on a TA Q5000 IR (TA Instruments, New Castle, DE, USA) in a nitrogen atmosphere $(40 \mathrm{~mL} / \mathrm{min})$, the samples being heated from room temperature to $700^{\circ} \mathrm{C}$ with a $10^{\circ} \mathrm{C} / \mathrm{min}$ heating rate.

\subsection{Statistical Analysis}

The swelling experiments were performed in duplicate, and the results are presented as average values and standard deviation. Student's $t$-test was used for assessment of statistical significance. Values were considered significant at $p<0.05$.

\section{Results and Discussion}

The studied hydrogels were obtained by the radical copolymerization of MAA with BIS in aqueous solution, in the presence of APS as the initiator, by using different concentrations of the monomer, crosslinking agent, and MMT, within the usual concentration range employed for the synthesis of hydrogels. The standard hydrogel (SH), against which the concentration of the monomer and BIS was modified, while MMT was added in the case of composite hydrogels, contained $10 \mathrm{wt} . \%$ MAA and $2 \mathrm{~mol} \%$ BIS. For a better comparison of the effect of the various components, the viscoelastic properties were measured on asprepared hydrogels, whose composition was identical to the one of the precursor solution. Further, for a correct interpretation of the results, the water absorption/swelling degree was determined after the purification of the hydrogels and calculated in relation only to the amount of polymer in the hydrogel, excluding the amount of MMT incorporated in the case of composite hydrogels and taking into account the total monomer conversion. Because both the viscoelastic properties of hydrogels and their swelling degree depend on the monomer conversion, this was determined in each case, resulting very high conversions (90-98\%), which allows the comparison of rheological and swelling measurements in all cases. To prove the incorporation of the reinforcing agent, the purified composite hydrogels were further characterized by FT-IR spectroscopy, XRD, and TGA analyses.

\subsection{Influence of the Monomer Concentration}

The change in the monomer concentration in the initial mixture influences the crosslinking degree $[7,8,26]$ and the proportion of chain entanglements [26] within the hydrogels, and therefore their viscoelastic properties and swelling degree. 
The effect of the monomer concentration was studied for three different values, namely, $10,20,30 \mathrm{wt} \%$ based on the whole reaction mass by keeping constant the crosslinking agent (BIS, $2 \mathrm{~mol} \%$ to MAA) and the initiator (KPS $1 \mathrm{~mol} \%$ to MAA) concentrations.

\subsubsection{Viscoelastic Properties}

The obtained hydrogels in their as-prepared state were characterized by both amplitude sweep and frequency sweep measurements. The amplitude sweep measurements (Figure 1a) showed that the linear viscoelasticity region (LVER) was wider at lower monomer concentrations, indicating a higher elasticity of the hydrogel at lower monomer concentrations, while the frequency sweep measurements (Figure 1b) demonstrated the crosslinked character of the hydrogels as indicated by the storage modulus $\left(\mathrm{G}^{\prime}\right)$ being larger than the loss modulus $\left(G^{\prime \prime}\right)$ over the entire frequency range investigated [27]. The values of the two viscoelastic moduli increased with the monomer concentration (Figure 1b, Table 2), showing that a more rigid hydrogel was developed in the case of higher amounts of the monomer, which can be explained by a higher polymer content of the hydrogel, i.e., a lower swelling degree [22], a higher crosslinking density (Table 2), and a larger proportion of entangled polymer chains. The progressively more rigid hydrogels with a denser network, formed with increasing monomer concentration, displayed a 10 times increase in the $\mathrm{G}^{\prime}$ value at $1 \mathrm{~Hz}$ when doubling the monomer concentration, and a 30 times increase in $\mathrm{G}^{\prime}$ when the monomer concentration was tripled (Table 2). Further, the increasing dependence of $G^{\prime}$ and $G^{\prime \prime}$ on frequency indicated the presence of a network with large meshes in the case of all hydrogels due to the relatively low crosslinking agent/MAA mole ratio [27]. These results are in agreement with those of Mithra et al. [28], who investigated the viscoelastic properties of some sodium acrylate-based hydrogels with variable monomer contents. They similarly concluded that hydrogels with higher mechanical properties are obtained at higher monomer concentrations.

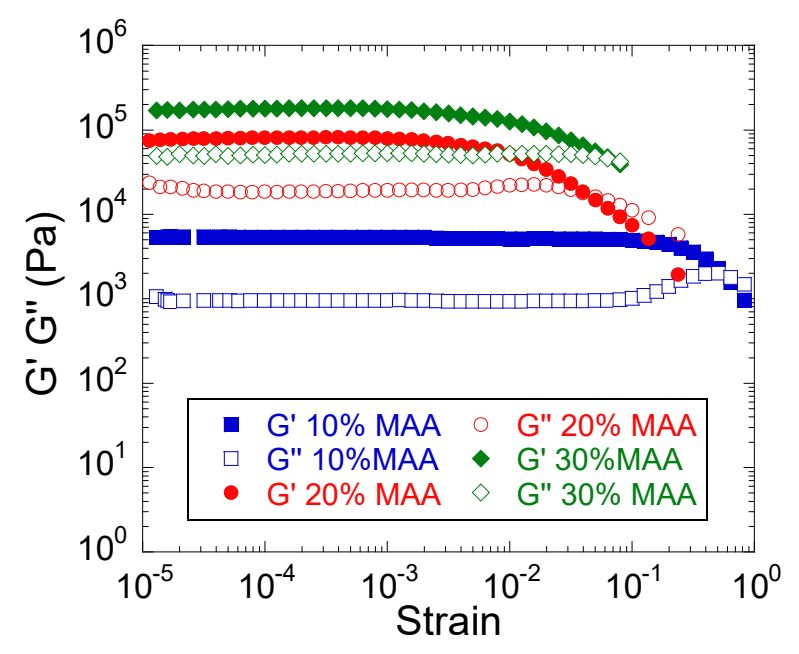

(a)

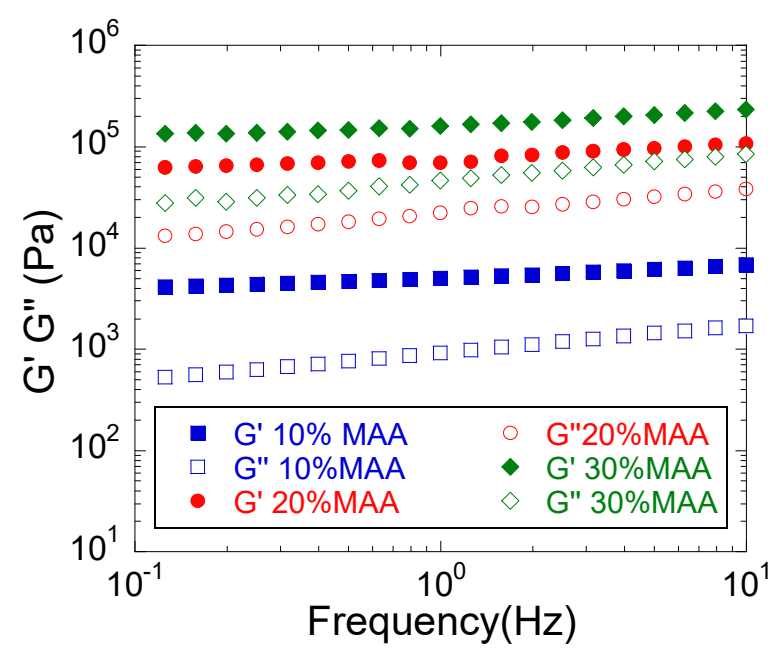

(b)

Figure 1. The influence of the monomer concentration on the viscoelastic properties of PMAA hydrogels (a) Amplitude sweep measurements (b) Frequency sweep measurements. Hydrogel composition: MAA, 2 mol\% BIS based on MAA, $0 \%$ MMT. 
Table 2. Dependence of the viscoelastic moduli (frequency sweep measurements) and some structural network parameters on the monomer concentration ${ }^{\mathbf{a}}$.

\begin{tabular}{ccccc}
\hline Sample Code & $\mathbf{G}^{\prime}(\mathbf{P a})^{\mathbf{b}}$ & $\mathbf{G}^{\prime \prime}(\mathbf{P a})^{\mathbf{b}}$ & $\overline{\mathbf{M}}_{\boldsymbol{c}} \mathbf{( \mathbf { D a } ) ^ { \mathbf { c } }}$ & $\boldsymbol{\rho}_{\boldsymbol{c}} \times 10^{-4}\left(\mathbf{m o l} / \mathbf{c m}^{3}\right)^{\mathbf{d}}$ \\
\hline SH & $5.05 \times 10^{3}$ & $0.92 \times 10^{3}$ & 874.9 & 14.7 \\
H20\% MAA & $53.8 \times 10^{3}$ & $13.3 \times 10^{3}$ & 368.7 & 35.0 \\
H30\% MAA & $160 \times 10^{3}$ & $46 \times 10^{3}$ & 193.8 & 66.6 \\
\hline
\end{tabular}

a Hydrogel composition: MAA, 2 mol\% BIS based on MAA, $0 \%$ MMT; ${ }^{b} \mathrm{G}^{\prime}$ and $\mathrm{G}^{\prime \prime}$ at $1 \mathrm{~Hz}$ obtained during the frequency sweep analyses; ${ }^{\mathrm{c}}$ Average molecular weight between crosslinks at $\mathrm{pH}$ 1.2, calculated with Equation (3); ${ }^{\mathrm{d}}$ Crosslinking density at $\mathrm{pH} 1.2$, calculated with Equation (5); ${ }^{\text {e }} \mathrm{SH}: 10 \% \mathrm{MAA}, 2 \mathrm{~mol} \% \mathrm{BIS}$ based on MAA, $0 \% \mathrm{MMT}$.

\subsubsection{Swelling Degree}

In the case of environmentally sensitive hydrogels, the swelling degree may be influenced by the medium in which the swelling takes place. PMAA hydrogels belong to the $\mathrm{pH}$ sensitive hydrogel category due to the presence of acidic groups within their molecules. When the $\mathrm{pH}$ value of the swelling medium is higher than $\mathrm{pKa}$, ionization of the acid groups in the hydrogel takes place, leading to the increase in the swelling degree [11]. The equilibrium swelling degree (ESD) of the hydrogels with different MAA contents was determined under various $\mathrm{pH}$ conditions $(1.2 ; 5.4 ; 7.4)$ by SD measurements of the swelling degree as a function of time at $37^{\circ} \mathrm{C}$ (Figure 2). The SD-time plots at $\mathrm{pH} 5.4$ showed that swelling was fast, the equilibrium value being reached after less than $60 \mathrm{~min}$ (Figure 2a). However, the $10 \mathrm{wt} . \%$ monomer hydrogels were fragile and difficult to handle, and as a consequence, we decided to compare the swelling degree of the samples at $72 \mathrm{~h}$ (Figure $2 \mathrm{~b}$ ), a time interval sufficient to reach ESD according to the experiment mentioned above.

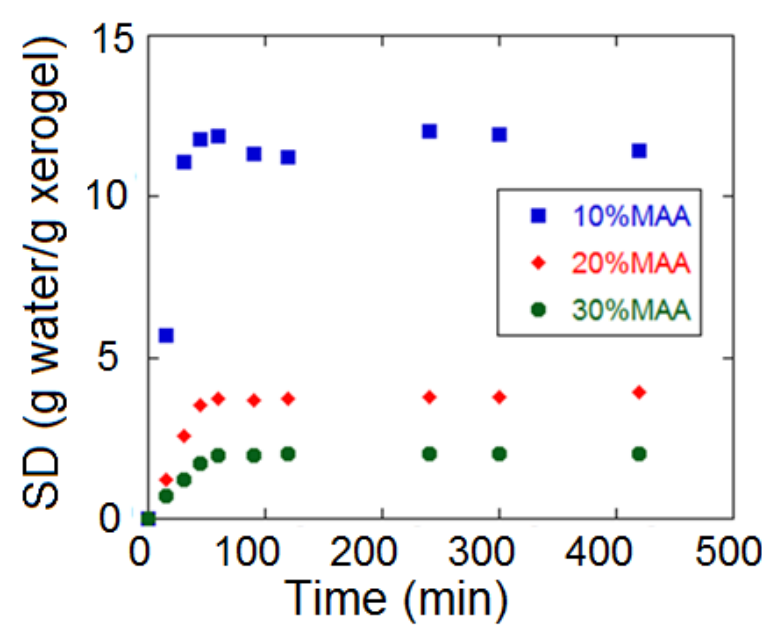

(a)

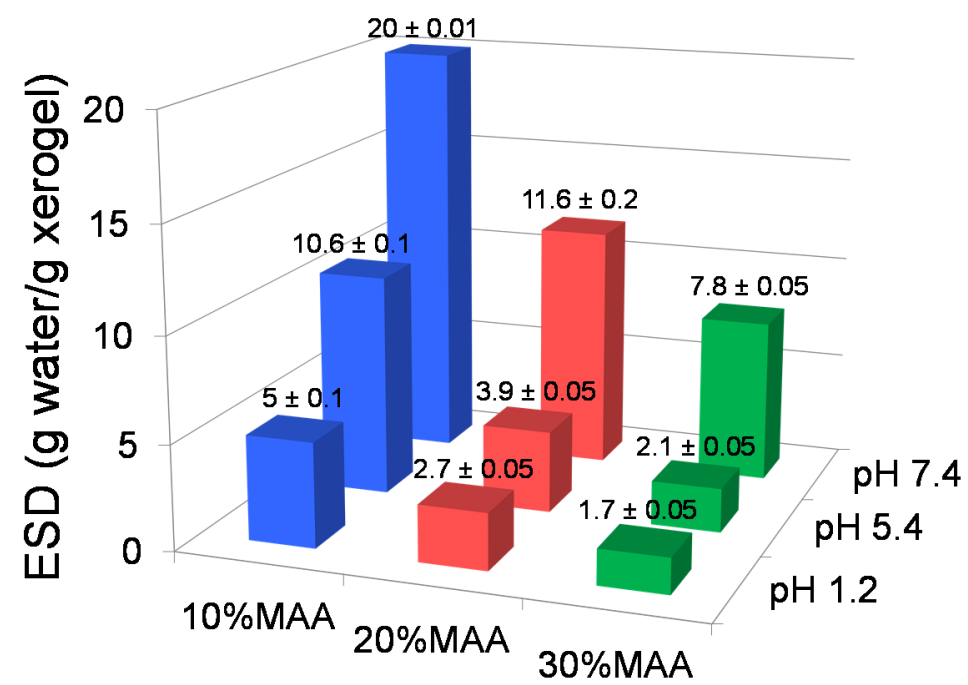

(b)

Figure 2. The influence of the monomer concentration on the swelling properties of hydrogels (a) Swelling curves at pH 5.4; (b) The effect of the monomer concentration on the swelling degree $(72 \mathrm{~h})$ at different $\mathrm{pH}$ values $(1.2 ; 5.4 ; 7.4)$. The results express average values \pm standard errors. Hydrogel composition: MAA, 2 mol $\%$ BIS based on MAA, $\%$ MMT.

As we expected based on the $\mathrm{pH}$-sensitive character of the hydrogels, regardless of the monomer concentration in the synthesis step, ESD increased with increasing $\mathrm{pH}$, with the highest swelling degree being obtained at pH 7.4 (Figure 2b, Supplementary Material Figure S1a). The higher the $\mathrm{pH}$ of the medium, the higher the proportion of ionized $\mathrm{COOH}$ groups in the hydrogel, leading to higher electrostatic repulsions and an increase in the osmotic pressure in the hydrogel, both of them causing a higher water absorption at equilibrium. The increase in $\mathrm{pH}$ from 1.2 to 7.4 led to a significant increase in ESD for all three types of hydrogels $(p<0.05)$, namely, approximately four times in 
the case of the hydrogel synthesized at 10\% MAA, 4.3 times for the one with $20 \mathrm{wt} \%$ MAA, and 4.6 times in the case of the $30 \mathrm{wt} . \%$ MAA hydrogel. Regardless of the $\mathrm{pH}$ value of the medium, the swelling degree increased with the decrease in the monomer concentration, in agreement with previous reports $[7,15,21,25,26]$. This can be explained by the decrease in the crosslinking density (Table 2), as a consequence of both a lower proportion of chain entanglements at lower monomer concentrations (Figure 2b) and the covalent crosslinking bridges being partially replaced by loops, resulting in intramolecular cyclization $[7,15,21,25]$. Tripling of the monomer concentration during synthesis led to a decrease in ESD of about 2.9 times at $\mathrm{pH}$ 1.2, five times at $\mathrm{pH}$ 5.4, and 2.6 times at $\mathrm{pH} 7.4$. Therefore, it can be stated that the three types of hydrogels do not behave very differently from this point of view either (Supplementary Material Figure S2).

The crosslinking density, determined in the case of hydrogels that swelled at $\mathrm{pH} 1.2$ (Table 2), displayed a strong increase with monomer concentration, because of the reasons shown above. In addition, the low value of $\overline{\mathrm{M}}_{\mathrm{c}}$ seems to indicate the formation of supplementary bridges through hydrophobic interactions among PMAA chains. The calculations showed that a 3-fold increase in the monomer concentration led to a 4.5 -fold enhancement of the crosslinking density at this $\mathrm{pH}$ value.

\subsection{Influence of the Crosslinking Agent Concentration}

The concentration of the crosslinking agent in the hydrogel network is an important parameter to investigate because it directly affects the swelling degree and therefore the viscoelastic properties of the hydrogel. In this study, the effect of three different concentrations of the crosslinking agent was investigated, namely, 1,2 and $3 \mathrm{~mol} \%$ relative to the monomer, with the concentration of the monomer being $10 \mathrm{wt} \%$ based on the whole hydrogel composition.

\subsubsection{Viscoelastic Properties}

The viscoelastic properties of hydrogels depend on their swelling degree [23] and therefore, to study the intrinsic effect of crosslinking agent concentration, measurements were made immediately after the synthesis of hydrogels, when the swelling degree was practically the same in all cases, dictated by the initial composition of the hydrogel.

The use of a higher concentration of crosslinking agent in the synthesis process leads to hydrogels with a higher density of crosslinking bridges, and therefore, more crosslinked structures are obtained that have higher mechanical properties. Indeed, the amplitude sweep and frequency sweep rheological measurements (Figure 3) showed that the hydrogels synthesized at a higher concentration of BIS were more rigid, displaying higher $\mathrm{G}^{\prime}$ values, in agreement with the expected formation of a more crosslinked network. Furthermore, the viscoelastic properties remained constant over a larger deformation range when the BIS concentration was lower, indicating a higher elasticity of the less crosslinked hydrogels (Figure 3a). Further, the frequency sweep measurements (Figure 3b) highlighted $\mathrm{G}^{\prime}>\mathrm{G}^{\prime \prime}$ over the entire frequency range studied, thus confirming the crosslinked character of the hydrogels [25]. The difference between $G^{\prime}$ and $G^{\prime \prime}$ increased with decreasing frequency, indicating the presence of a wide-mesh network [22], in agreement with the BIS concentration used. Table 3 shows the values of the parameters $\mathrm{G}^{\prime}$ and $\mathrm{G}^{\prime \prime}$ at $1 \mathrm{~Hz}$ frequency for the three crosslinking agent concentrations employed ( $1 \mathrm{~mole} \%, 2 \mathrm{~mol} \%$ and $3 \mathrm{~mol} \%$ relative to the monomer), and it can be noticed that the doubling of the BIS concentration ( $2 \mathrm{~mol} \%$ vs. 1 mole $\%$ ) led to a doubling of $\mathrm{G}^{\prime}$, while tripling the crosslinking agent concentration ( $3 \mathrm{~mol} \%$ vs. 1 mole\%) caused a $\mathrm{G}^{\prime}$ increase of about fivefold. By comparison, the increase in the monomer concentration led to a much greater increase in $\mathrm{G}^{\prime}$ (Table 2), i.e., a 2-fold enhancement of the monomer concentration led to a 10-fold increase in $\mathrm{G}^{\prime}$, while tripling the monomer concentration resulted in an approximately 30 -fold increase in $\mathrm{G}^{\prime}$, an effect which can be ascribed to both the increase in the crosslinking degree and the higher polymer concentration in the swollen hydrogel, as mentioned above $[29,30]$. 


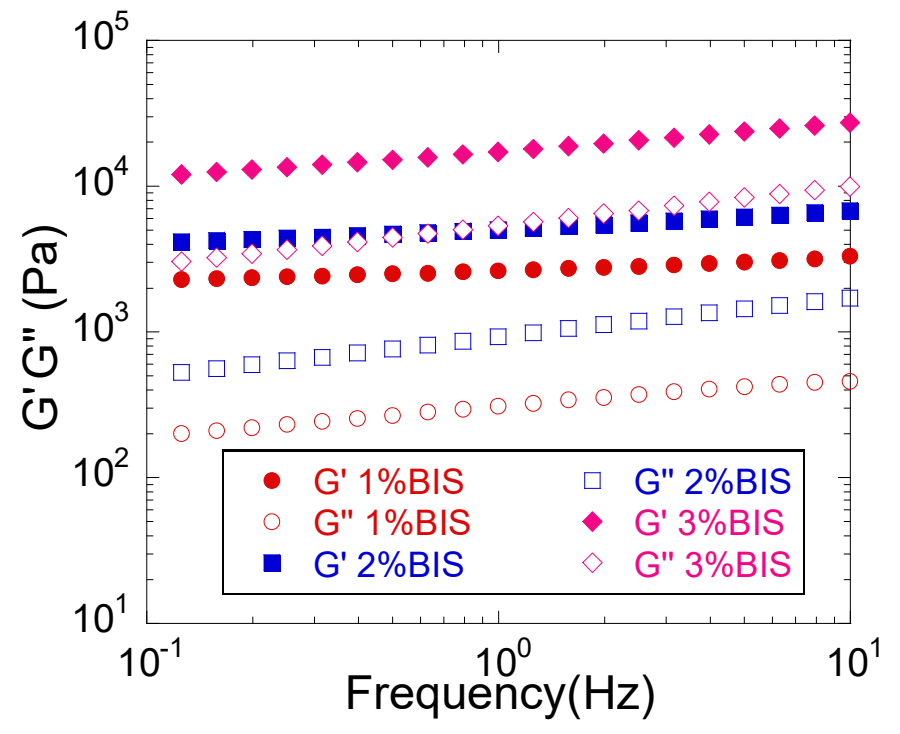

(a)

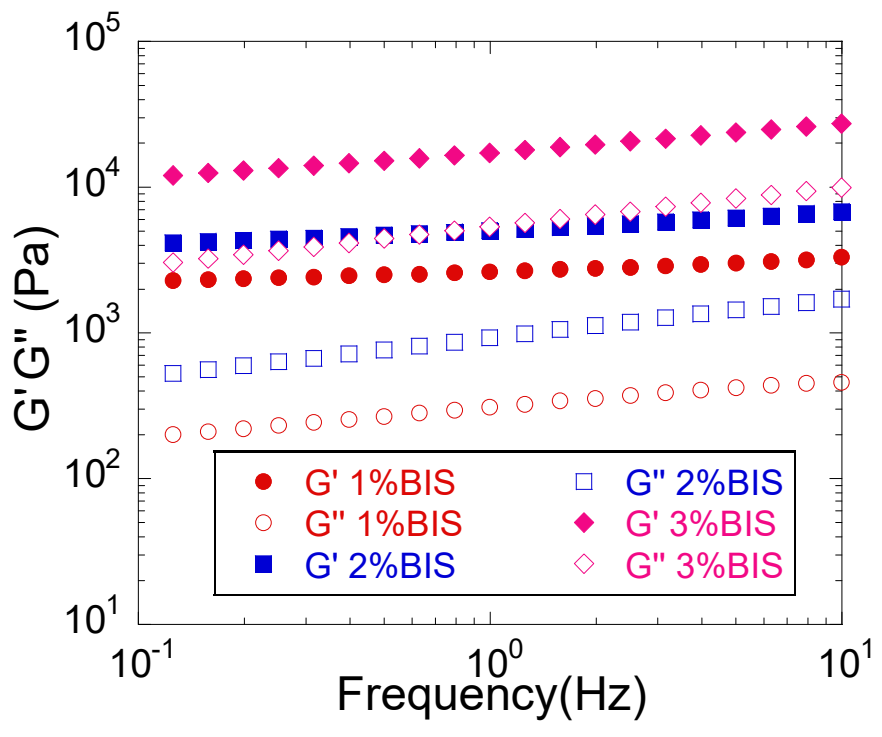

(b)

Figure 3. The influence of the crosslinking agent concentration on the viscoelastic properties of PMAA hydrogels (a) Amplitude sweep measurements (b) Frequency sweep measurements. Hydrogel composition: BIS, 10\% MAA based on the whole hydrogel composition, $0 \%$ MMT.

Table 3. Dependence of the viscoelastic moduli (frequency sweep measurements) and some structural network parameters on the crosslinking agent concentration ${ }^{\mathrm{a}}$.

\begin{tabular}{ccccc}
\hline Sample Code & $\mathbf{G}^{\prime}(\mathbf{P a})^{\mathbf{b}}$ & $\mathbf{G}^{\prime \prime}(\mathbf{P a})^{\mathbf{b}}$ & $\overline{\mathbf{M}}_{\boldsymbol{c}} \mathbf{( \mathbf { D a } ) ^ { \mathbf { c } }}$ & $\boldsymbol{\rho}_{\boldsymbol{c}} \times 10^{-4}\left(\mathbf{m o l} / \mathbf{c m}^{3}\right)^{\mathbf{d}}$ \\
\hline H1\%BIS & $2.63 \times 10^{3}$ & $0.31 \times 10^{3}$ & 915.2 & 14.1 \\
SH e $_{\text {H3\% BIS }}^{5.05 \times 10^{3}}$ & $0.92 \times 10^{3}$ & 874.9 & 14.7 \\
\hline
\end{tabular}

a Hydrogel composition: BIS, $10 \%$ MAA based on the whole hydrogel composition, $0 \%$ MMT; ${ }^{b} \mathrm{G}^{\prime}$ and $\mathrm{G}^{\prime \prime}$ at $1 \mathrm{~Hz}$ obtained during the frequency sweep analyses; ${ }^{\mathrm{c}}$ Average molecular weight between crosslinks at $\mathrm{pH} 1.2$, calculated with Equation (3); ${ }^{\mathrm{d}}$ Crosslinking density at $\mathrm{pH} 1.2$, calculated with Equation (5); ${ }^{\text {S }} \mathrm{SH}: 10 \% \mathrm{MAA}, 2 \mathrm{~mol} \% \mathrm{BIS}$ based on MAA, $0 \% \mathrm{MMT}$.

\subsubsection{Swelling Degree}

The adjustment of the crosslinking agent concentration is one of the most important methods of controlling the crosslinking density of a hydrogel and, as a consequence, of the ability to swell in water (Supplementary Material Figure S1a,b). Indeed, our experiments showed that by increasing the concentration of BIS, the swelling degree of the hydrogel decreased, regardless of the $\mathrm{pH}$ of the medium (Figure 4), due to a higher crosslinking density (Table 3). Tripling the BIS concentration resulted in a reduction in ESD of only 1.2 times at $\mathrm{pH} 1.2$, as compared to approximately 3.3 times and 2.5 times at $\mathrm{pH} 5.4$ and 7.4, respectively. In comparison with the hydrogels synthesized at various monomer concentrations, the effect of increasing BIS concentration on ESD had a relatively similar amplitude at $\mathrm{pH} 5.4$ and 7.4, but the behavior was very different at acidic $\mathrm{pH}$, when tripling the BIS concentration led to an insignificant decrease in ESD, as compared to a decrease of almost 3-fold in the previous case. This dissimilarity at $\mathrm{pH} 1.2$ is the consequence of the large difference between the influence of the two parameters upon the crosslinking density: a 3-fold increase in the monomer concentration led to a 4.5 increase in $\rho_{\mathrm{c}}$ as compared with only a 1.5-fold increase for the same increase in the crosslinking agent concentration. 


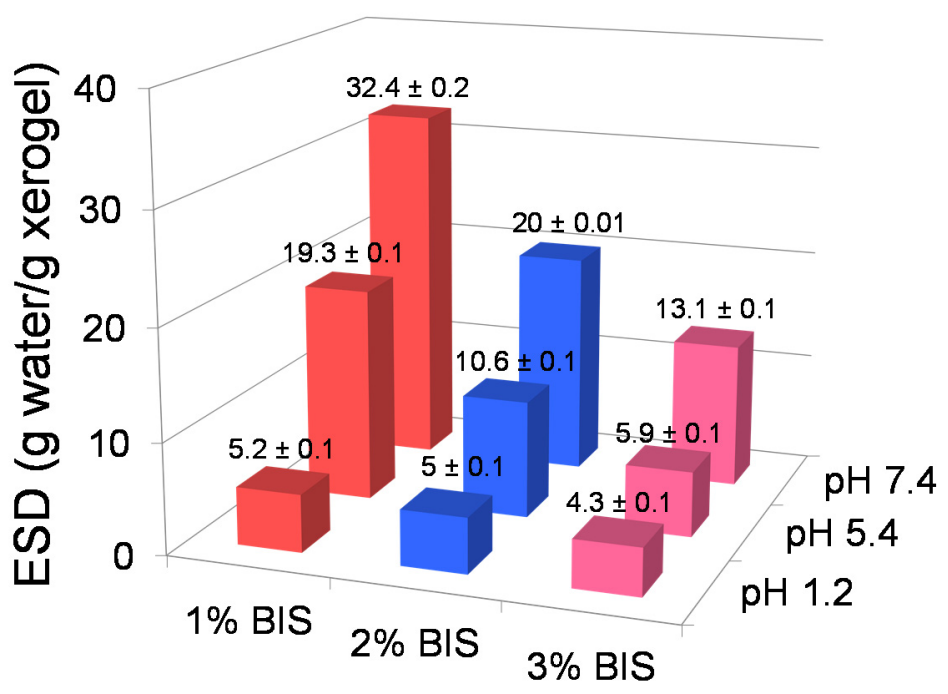

Figure 4. The influence of the crosslinking agent concentration on the swelling degree $(72 \mathrm{~h})$ at different $\mathrm{pH}$ values. The results are the average values \pm standard errors. Hydrogel composition: BIS, 10\% MAA based on the whole hydrogel composition, $0 \%$ MMT.

Regarding the effect of the $\mathrm{pH}$ of the medium, in this case as well, the $\mathrm{pH}$ increase led to an increase in ESD as expected; the reason for this effect is explained above. It is worth noting, however, that the two series of hydrogels behaved differently from this of point view as well. Namely, while in the case of hydrogels obtained at different monomer concentrations, the ratio $\mathrm{R}=(\mathrm{ESD}$ at $\mathrm{pH} 7.4) /(\mathrm{ESD}$ at $\mathrm{pH} 1.2)$ practically did not depend on the monomer concentration at synthesis $(R \approx 4)$, in the case of the crosslinking agent concentration modification, $\mathrm{R}$ significantly decreased with the increase in the amount of BIS from about 6 for the hydrogel with $1 \mathrm{~mol} \%$ BIS relative to the monomer, to 4 and about 3 in the case of hydrogels with $2 \mathrm{~mol} \%$ BIS and $3 \mathrm{~mol} \%$ BIS, respectively $(p<0.05)$. We do not have an explanation for this phenomenon at this time.

\subsection{Influence of the Reinforcing Agent}

This chapter describes the influence of the reinforcing agent concentration, which can affect the viscoelastic and swelling behaviors of the hydrogel together with monomer and crosslinking agent concentrations. Hydrogels with $10 \mathrm{wt} \%$ monomer in the precursor solution were analyzed, with the concentration of the crosslinking agent being $2 \mathrm{~mol} \%$ relative to the monomer. The concentrations of the investigated reinforcing agent were 1,2, $3 \mathrm{wt} \%$ based on the whole mass of hydrogel, i.e., 9.65, 19.3, $28.95 \mathrm{wt} \%$ in comparison to the amount of monomers (Table 1). The composite hydrogels obtained were structurally characterized by FT-IR, TGA, and XRD analyses to highlight the presence of the reinforcing agent, and the rheological properties and water absorption in solutions with various $\mathrm{pH}$ values were determined. We performed SEM analyses for a representative sample (H2\%MMT) (Supplementary Material Figure S3).

\subsubsection{FT-IR Analyses}

FT-IR spectroscopy was used to prove the clay inclusion within the PMAA matrix and to detect whether interactions between components occurred. The FT-IR spectra of the composite xerogels displayed the characteristic peaks of the stretching vibrations of the $\mathrm{Si}-\mathrm{O}-\mathrm{Si}$ and $\mathrm{Si}-\mathrm{O}-\mathrm{Al}$ groups in MMT, thus confirming its presence in the material (Figure 5). We also noticed the increase in the intensity of the MMT characteristic peak in accordance with the amount of reinforcing agent introduced in the synthesis. In comparison to neat MMT, the characteristic peak of the clay at $999 \mathrm{~cm}^{-1}$ shifted to higher wavenumbers in the composite xerogels, indicating the presence of interactions between the clay layers and the PMAA network (Table 4). Further, the values of the peaks characteristic of the 
carboxylic group bonds $(\mathrm{C}=\mathrm{O}, \mathrm{C}-\mathrm{O})$ underwent shifts to higher wavenumbers in the case of the composite xerogels obtained, which reinforces the above statement.

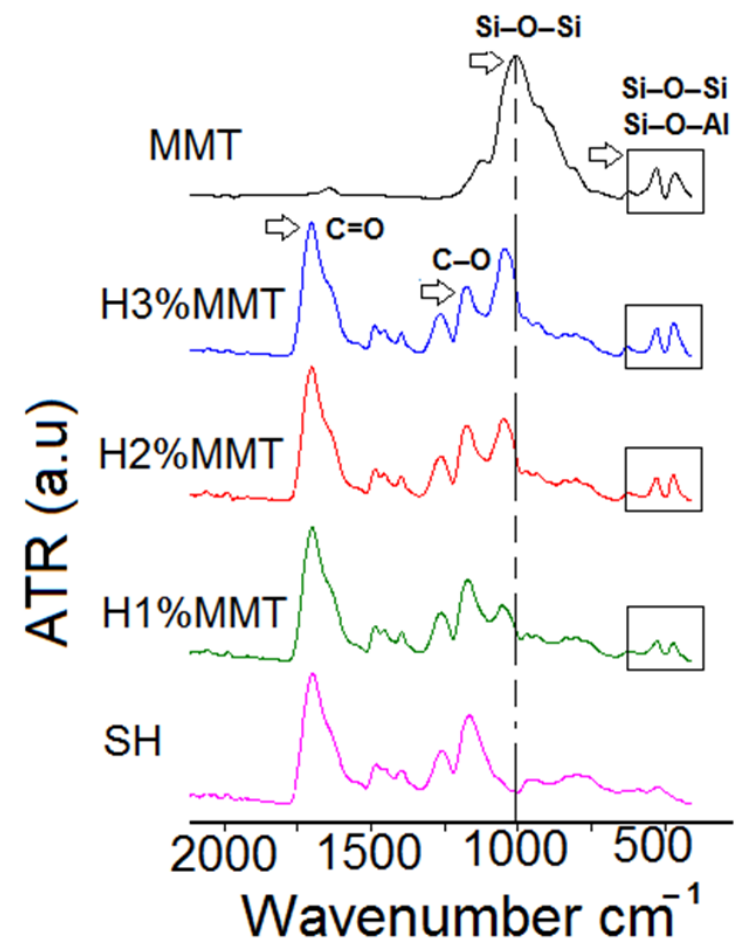

Figure 5. FT-IR spectra of the composite xerogels investigated and MMT. SH: 10\%MAA, 2 mol\%BIS based on MAA, $0 \%$ MMT.

Table 4. Characteristic FT-IR peak values of the investigated samples ${ }^{\mathrm{a}}$.

\begin{tabular}{ccc}
\hline Sample Code & PMAA $\left.\mathbf{( c m}^{-\mathbf{1}}\right)$ & MMT $\left.\mathbf{( c m}^{-\mathbf{1}}\right)$ \\
\hline MMT & - & 999 \\
SH & $1692 ; 1158$ & - \\
H1\%MMT & $1693 ; 1164$ & 1046 \\
H2\%MMT & $1695 ; 1167$ & 1042 \\
H3\%MMT & $1693 ; 1168$ & 1039
\end{tabular}

a Hydrogel composition: MMT, 10\% MAA based on the whole hydrogel composition, 2 mol\%BIS based on MAA b SH: 10\%MAA, 2 mol\%BIS based on MAA, $0 \%$ MMT.

\subsubsection{XRD Analyses}

The X-ray diffractograms (XRD) obtained for the studied composites and the pure MMT are displayed in Figure 6. By monitoring the position (20), shape, and intensity of the clay characteristic diffraction line in the composite structure, the intercalation/exfoliation phenomenon can be highlighted. In the case of $\mathrm{SH}$, a peak characteristic of PMAA $\left(2 \theta=15^{\circ}\right)$ was observed, which suggests the crystallinity of the polymer used in the synthesis of the hydrogel [19]. Once the MMT content of the xerogel increased, the intensity of this peak decreased, indicating the decrease in the crystallinity degree [31]. The analyses showed that in all cases, the peaks shifted to lower values (Table 5), which means that the d-spacing increased, suggesting that the clay layers were intercalated between the polymer chains. 


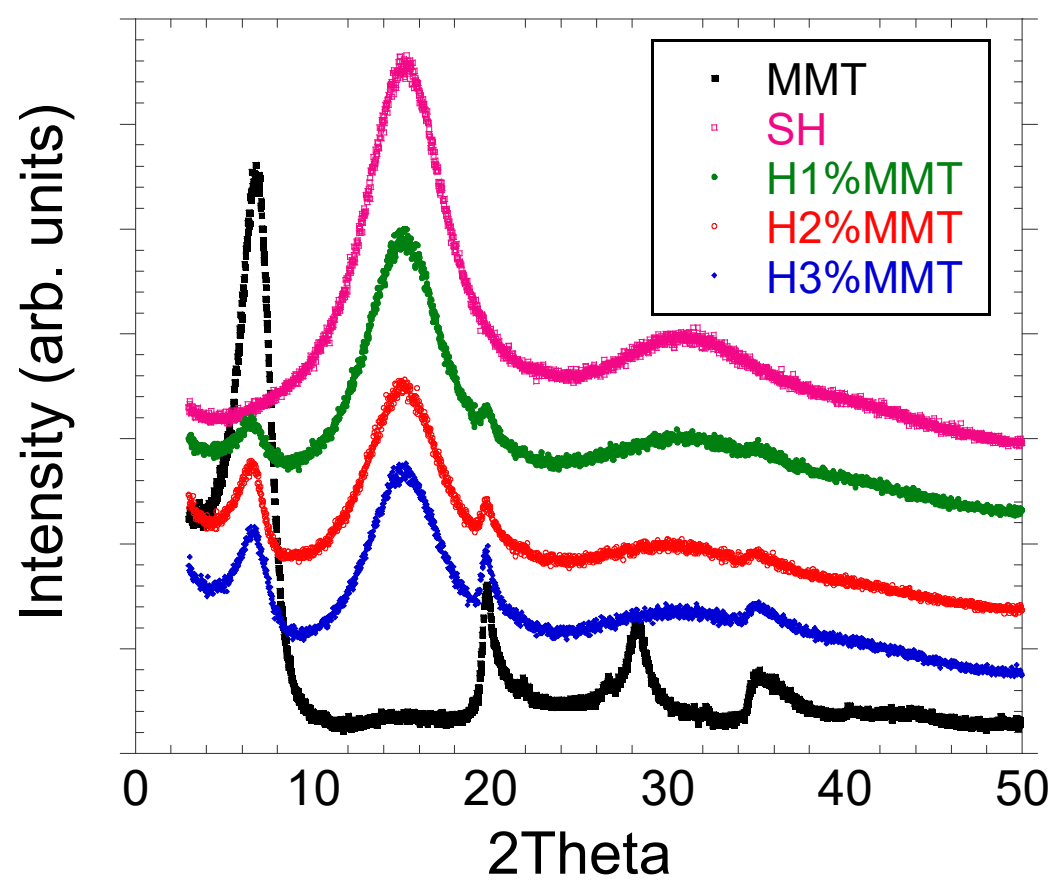

Figure 6. The diffractograms of MMT and the xerogels investigated. SH: 10\%MAA, 2 mol\%BIS based on MAA, $0 \%$ MMT.

Table 5. The XRD values obtained for the investigated xerogels ${ }^{a}$.

\begin{tabular}{ccc}
\hline Sample Code & 2 Theta (̊̊) & d-Spacing (nm) \\
\hline MMT & 6.8 & 13 \\
H1\%MMT & 6.63 & 13.3 \\
H2\%MMT & 6.5 & 13.5 \\
H3\%MMT & 6.7 & 13.2 \\
\hline
\end{tabular}

a Xerogel composition: MMT, 10\% MAA based on the whole hydrogel composition, $2 \mathrm{~mol} \%$ BIS based on MAA.

\subsubsection{Thermogravimetric Analyses}

The thermogravimetric analysis revealed the presence of three decomposition stages in the case of the nanocomposite hydrogels (Table 6, Figure 7). The first stage of decomposition that took place up to $120^{\circ} \mathrm{C}$ was insignificant and can be attributed to the volatilization of the network water [12] still existing in the hydrogels.

Table 6. The TGA/DTG results obtained for investigated composite hydrogels ${ }^{\text {a }}$.

\begin{tabular}{|c|c|c|c|c|c|c|}
\hline \multirow{2}{*}{ Sample Code } & \multicolumn{3}{|c|}{ Weight Loss Intervals (\%) } & \multicolumn{2}{|c|}{ Decomposition Temperatures $\left({ }^{\circ} \mathrm{C}\right)$} & \multirow{2}{*}{$\begin{array}{c}\text { Residue (\%) at } \\
700{ }^{\circ} \mathrm{C}\end{array}$} \\
\hline & $0-120{ }^{\circ} \mathrm{C}$ & $120-300{ }^{\circ} \mathrm{C}$ & $300-700{ }^{\circ} \mathrm{C}$ & $\mathbf{T}_{1}$ & $\mathbf{T}_{2}$ & \\
\hline $\mathrm{SH}^{\mathrm{b}}$ & 2.87 & 18.96 & 71.9 & 225 & 424.5 & 6.31 \\
\hline $\mathrm{H} 1 \% \mathrm{MMT}$ & 3.22 & 18.14 & 66.35 & 222.2 & 411.6 & 12.27 \\
\hline $\mathrm{H} 2 \% \mathrm{MMT}$ & 3.44 & 17.24 & 59.40 & 221.3 & 401.9 & 19.90 \\
\hline H3\%MMT & 3.173 & 16.44 & 55.16 & 220.5 & 393.2 & 25.26 \\
\hline
\end{tabular}

${ }^{a}$ Hydrogel composition: MMT, 10\% MAA based on the whole hydrogel composition, 2 mol\%BIS based on MAA. ${ }^{b}$ SH: $10 \%$ MAA, $2 \mathrm{~mol} \%$ BIS based on MAA, $0 \%$ MMT. 


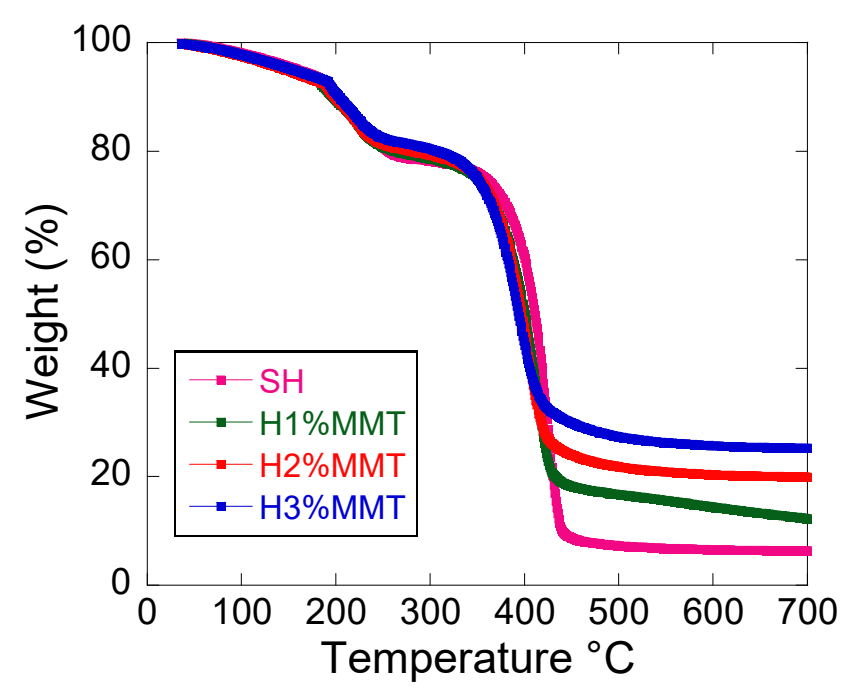

(a)

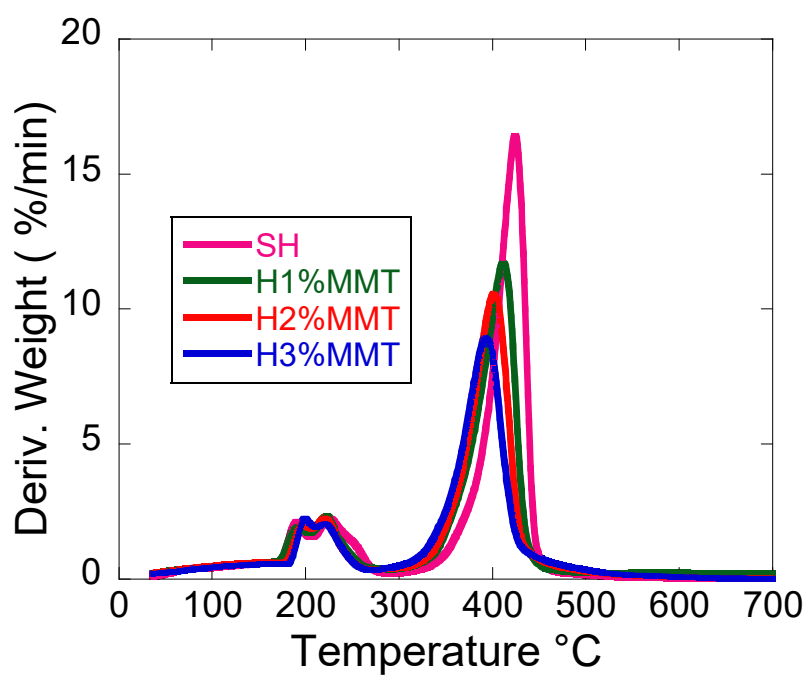

(b)

Figure 7. TGA (a) and TDG (b) curves of the obtained composite hydrogels. SH: 10\%MAA, 2 mol\%BIS based on MAA, $0 \%$ MMT.

In the second stage $\left(120-300{ }^{\circ} \mathrm{C}\right)$, xerogel dehydration occurred by inter- and intramolecular elimination of water between $\mathrm{COOH}$ groups with the formation of anhydride cycles, as well as decarboxylation of $\mathrm{COOH}$ groups and $\mathrm{CO}_{2}$ elimination [27]. The last stage $\left(300-700^{\circ} \mathrm{C}\right)$ is ascribed to the total decomposition of the PMAA chains [28]. Total loss of mass was reduced in the case of composite hydrogels due to the presence of MMT that does not degrade at these temperatures. The residue value obtained by TGA analyses is often employed to demonstrate the presence and/or quantify the amount of inorganic agents used to reinforce polymers [29]. All MMT-containing samples showed a higher residue than the control sample at $700{ }^{\circ} \mathrm{C}$, which increased with increasing MMT amount in the sample (Table 6). The residue amount was in good agreement with the initial percentage of MMT in the hydrogels (Table 1). The data in Table 6 also show that when the MMT content increased, $\mathrm{T}_{2}$ decreased. This phenomenon may be explained by a lower degree of crystallinity of the polymer at higher MMT concentrations (see XRD analyses), leading to a smaller decomposition temperature of the PMAA chains.

\subsubsection{Viscoelastic Properties}

As in the case of the unreinforced hydrogels, the viscoelastic properties were investigated immediately after synthesis. Amplitude sweep measurements (Figure 8a) showed that even in the case of composite hydrogels, the viscoelastic properties were kept constant over a wide range of deformation $\left(10^{-5}-10^{-1}\right)$, and therefore no appreciable decrease in elasticity (narrowing of LVER) with increasing amount of MMT was noticed. The frequency sweep measurements (Figure 8b, Table 7) showed that $G^{\prime}$ was higher than $G^{\prime \prime}$ over the whole frequency range investigated, thus demonstrating the crosslinked character of the hydrogels [32-34].

The introduction of MMT into hydrogels led to an improvement of their mechanical properties, a fact demonstrated by the increase in the values of the viscoelastic modules with increasing clay concentration. For a $10 \mathrm{wt} . \%$ initial monomer concentration, $\mathrm{G}^{\prime}$ was approximately two times higher when $3 \mathrm{wt} \%$ MMT was used as compared to the unreinforced hydrogel (Figure 9). However, tripling the amount of the reinforcing agent in the hydrogel (from $1 \mathrm{wt} . \%$ to $3 \mathrm{wt} . \%$ ) led to a relatively small increase in $\mathrm{G}^{\prime}$ of about 1.5 times (from $8.8 \times 10^{3} \mathrm{~Pa}$ to $12.4 \times 10^{3} \mathrm{~Pa}$, Table 7), much lower in comparison to the increase in $G^{\prime}$ when the monomer concentration $(\approx 30$ times, Table 2$)$ or crosslinking agent $(\approx$ six times, Table 3$)$ tripled, which seems to indicate that the existing interaction between the clay and the network was weak in the presence of water. 


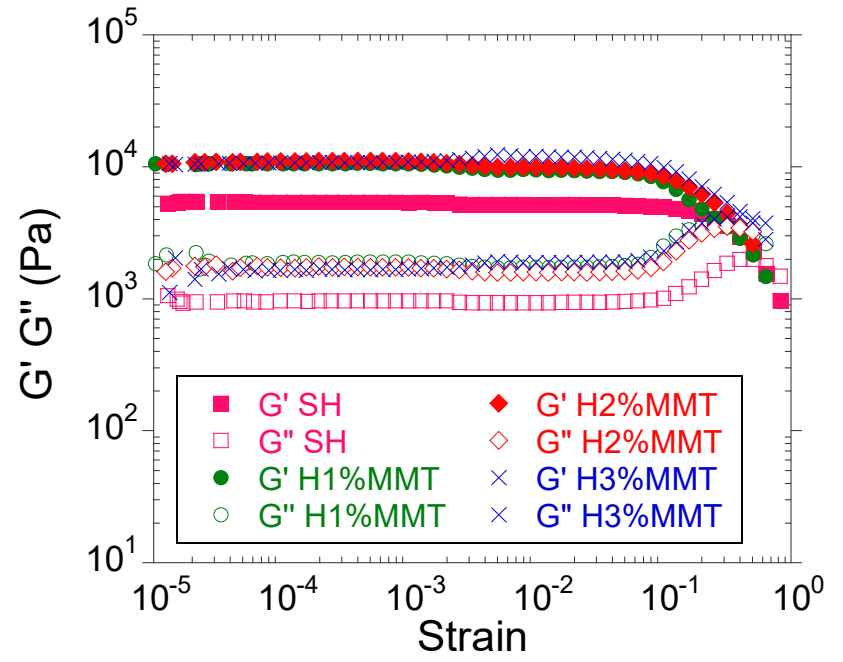

(a)

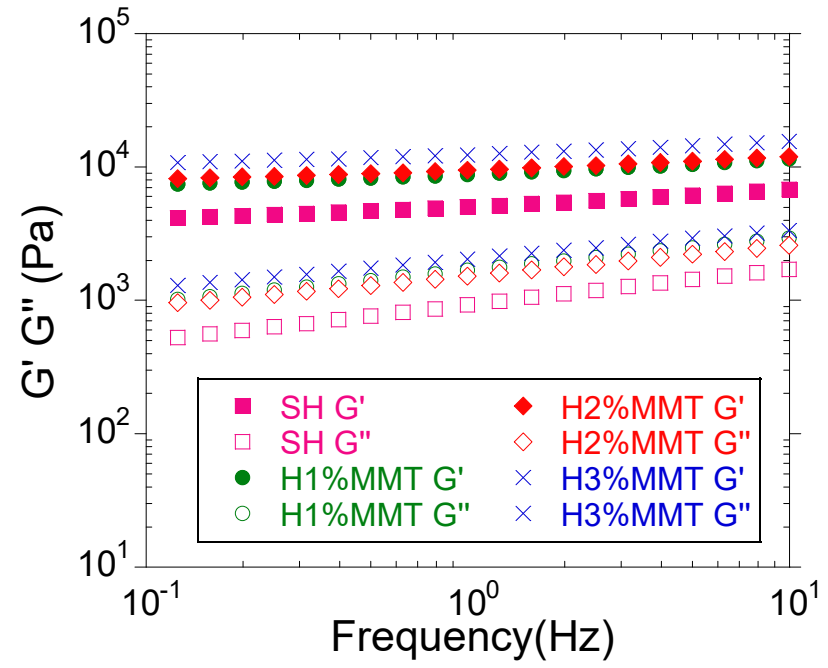

(b)

Figure 8. The influence of the MMT concentration on the viscoelastic properties of PMAA hydrogels (a) Amplitude sweep measurements (b) Frequency sweep measurements. SH: 10\%MAA, 2 mol\%BIS based on MAA, 0\%MMT.

Table 7. Dependence of the viscoelastic moduli (frequency sweep measurements) and some structural network parameters on MMT concentration ${ }^{\mathrm{a}}$.

\begin{tabular}{ccccc}
\hline Sample Code & $\mathbf{G}^{\prime}(\mathbf{P a})^{\mathbf{b}}$ & $\mathbf{G}^{\prime \prime}(\mathbf{P a})^{\mathbf{b}}$ & $\overline{\mathbf{M}}_{\mathbf{c}}(\mathbf{D a})^{\mathbf{c}}$ & $\boldsymbol{\rho}_{\boldsymbol{c}} \times 10^{-4}\left(\mathbf{m o l} / \mathbf{c m}^{3}\right)^{\mathbf{d}}$ \\
\hline SH & $5.05 \times 10^{3}$ & $0.93 \times 10^{3}$ & 874.9 & 14.7 \\
H1\%MMT & $8.8 \times 10^{3}$ & $1.67 \times 10^{3}$ & 904.3 & 14.3 \\
H2\%MMT & $9.5 \times 10^{3}$ & $1.52 \times 10^{3}$ & 774.0 & 16.7 \\
H3\%MMT & $12.4 \times 10^{3}$ & $2.04 \times 10^{3}$ & 689.5 & 18.7 \\
\hline
\end{tabular}

a Hydrogel composition: MMT, 10\% MAA based on the whole hydrogel composition, 2 mol\%BIS based on MAA; ${ }^{\mathrm{b}} \mathrm{G}^{\prime}$ and G" at $1 \mathrm{~Hz}$ obtained during the frequency sweep analyses; ${ }^{\mathrm{c}}$ Average molecular weight between crosslinks at $\mathrm{pH} 1.2$, calculated with Equation (3);

${ }^{\mathrm{d}}$ Crosslinking density at $\mathrm{pH}$ 1.2, calculated with Equation (5); ${ }^{\mathrm{e}} \mathrm{SH}: 10 \% \mathrm{MAA}, 2 \mathrm{~mol} \% \mathrm{BIS}$ based on MAA, 0\%MMT.

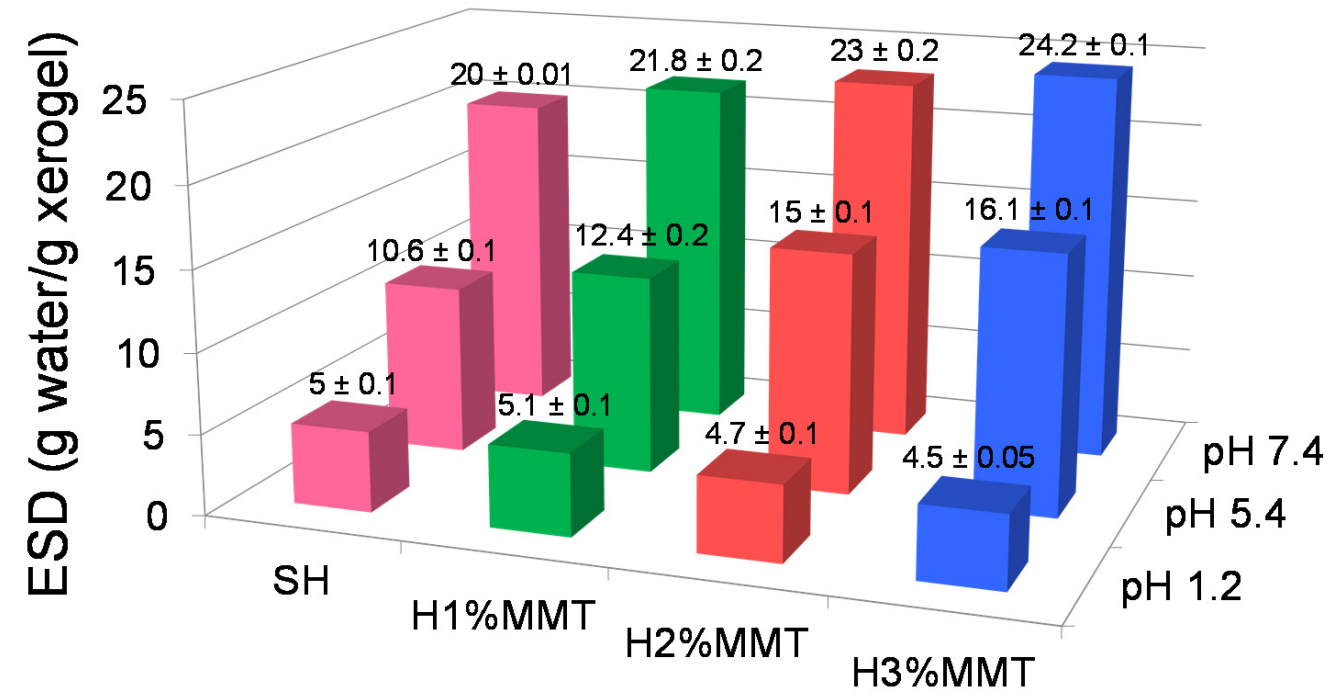

Figure 9. The influence of the reinforcing agent on the swelling degree $(72 \mathrm{~h})$ at different $\mathrm{pH}$ values. Results express average values \pm standard errors. $\mathrm{SH}$ : 10\%MAA, 2 mol\%BIS based on MAA, $\%$ MMT. 


\subsubsection{Swelling Degree}

The influence of the amount of MMT on the swelling degree of the composite hydrogels was studied at $\mathrm{pH} 1.2,5.4$, and 7.4 (Figure 9). The results showed that the swelling degree significantly increased with increasing $\mathrm{pH}$, as expected $(p<0.05)$, in agreement with the $\mathrm{pH}$-sensitive character of these PMAA hydrogels (Supplementary Material Figure S1c). However, unlike the effect of the monomer and crosslinking agent concentrations, when larger amounts of these components led to a swelling decrease due to the formation of a more crosslinked network (Supplementary material Figure S2), increasing the concentration of MMT at pH 5.4 and 7.4 resulted in enhanced water absorption. This can be explained by the fact that MMT had very little influence on the crosslinking degree of the hydrogel (Table 7), but instead, because of its basic character (a $2 \mathrm{wt} \%$ MMT aqueous dispersion displayed a $\mathrm{pH}$ of 8.9), MMT led to the formation of carboxylate groups in the hydrogel during the synthesis stage, the proportion of which was higher for higher MMT concentrations. As a result, in ionizing media ( $\mathrm{pH} 5.4$ and 7.4), these already formed anionic charges added to the newly formed ones because of the environment, leading to a differentiation of hydrogels in terms of total charge. The higher the amount of MMT in the hydrogel, the higher the number of $\mathrm{COO}^{-}$groups, leading to a higher swelling degree. It is worth noting that the ionization of $\mathrm{COOH}$ groups with the formation of negatively charged polymer chains begins at a $\mathrm{pH}$ higher than 5 [11]. The intrinsic effect of MMT on the swelling degree could be observed at $\mathrm{pH} 1.2$, when, due to the strongly acidic medium, all carboxylate groups were transformed into carboxyl groups, and therefore the effect of MMT basicity was canceled [35]. The results showed that, at strongly acidic $\mathrm{pH}$, the increase in MMT concentration led to a slight decrease in ESD (1.1 times for a 3-fold increase in MMT concentration-Figure 9), as a consequence of the enhanced crosslinking density (Table 7), similar to the effect of increasing BIS concentration under the same environmental conditions. This seems to indicate a slight crosslinking effect of MMT, which can be seen only at low swelling degrees.

SEM analyses performed for a representative sample (H2\%MMT) swollen at three different $\mathrm{pH}$ values revealed that the hydrogels present large interconnected pores which change their size depending on the swelling medium (Supplementary Material Figure S3).

\section{Conclusions}

The present paper comparatively discusses, for the first time, the influence of the monomer (MAA), crosslinking agent (BIS), and reinforcing agent (MMT) concentrations employed within the usual range, upon the viscoelastic properties determined on the as-prepared hydrogels, and the water absorption/swelling degree at various $\mathrm{pH}$ values of the PMAA hydrogels. It should be mentioned that the two properties investigated here are essential in establishing the applications of hydrogels. The results showed that the most important effect on the viscoelastic properties was displayed by monomer concentration, the tripling of which led to an approximate 30-fold increase in $\mathrm{G}^{\prime}$ as compared to an increase of only five times in the case of BIS and 1.5 times when the MMT concentration was raised from $1 \mathrm{wt} \%$ to $3 \mathrm{wt} \%$ (Figure 10).

The swelling studies confirmed, as expected, the $\mathrm{pH}$-sensitive character of the studied hydrogels, the ESD increasing with the $\mathrm{pH}$ of the medium in all cases. The increase in the concentrations of the monomer and crosslinking agent led to a decrease in water absorption due to the increase in the crosslinking density of the hydrogels in both cases.

The amplitude of the effect of the monomer and BIS concentrations on water absorption was similar at $\mathrm{pH} 5.4$ and 7.4, but they behaved very differently at acidic $\mathrm{pH}$, when the effect of the monomer amount was much stronger. Further, the $\mathrm{pH}$ increase had different effects in the case of these two hydrogels series. Namely, while in the case of hydrogels obtained at different monomer concentrations, the (ESD at $\mathrm{pH}$ 7.4)/(ESD at $\mathrm{pH}$ 1.2) ratio practically did not depend on the amount of the monomer, the same ratio decreased with the crosslinking agent concentration. 


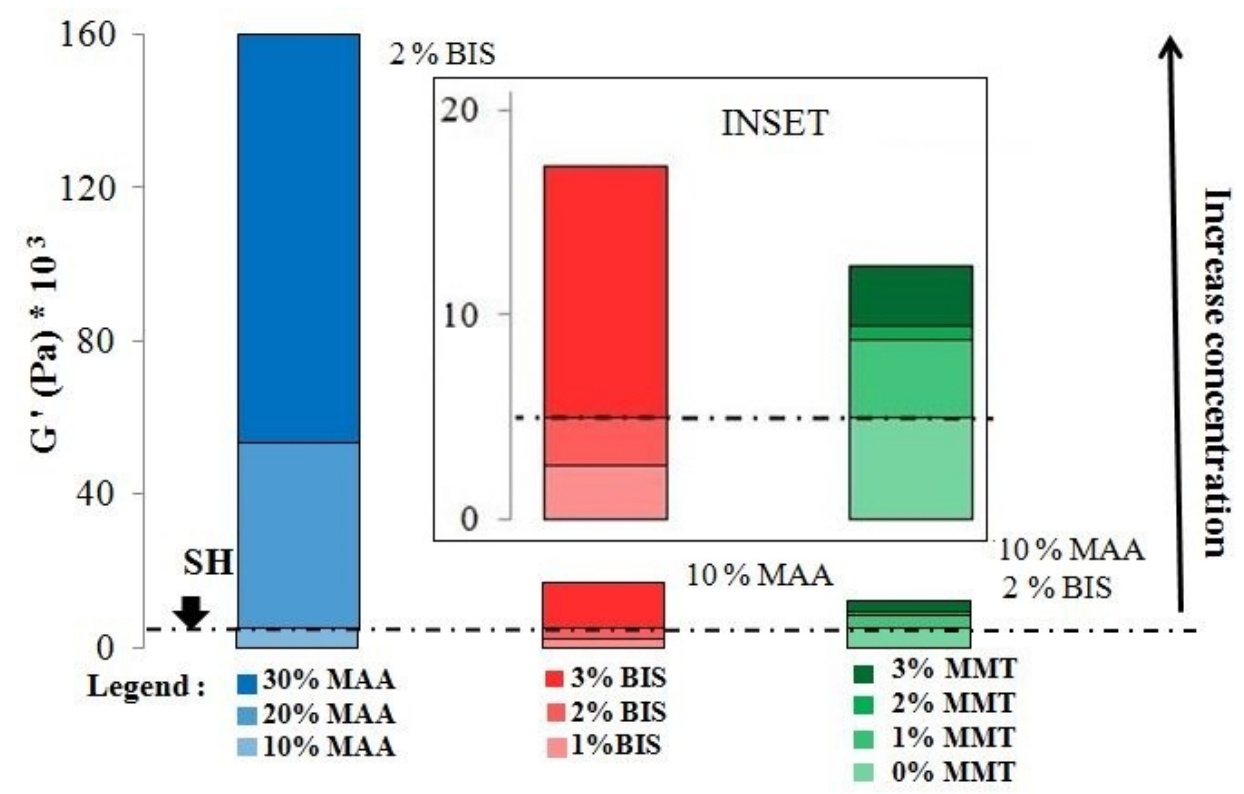

Figure 10. Comparative influence of the monomer, crosslinking agent, and reinforcing agent concentrations on the viscoelastic properties of PMAA hydrogels.

As opposed to the effects of the monomer and crosslinking agent concentrations, the increase in the MMT concentration in the hydrogel resulted in an increase in the swelling degree of the hydrogel at $\mathrm{pH} 5.4$ and 7.4, due to the basic character of the clay, while at $\mathrm{pH}$ 1.2 , a slight decrease in water absorption was noticed, ascribable to a slight crosslinking effect of the MMT, in agreement with the rheological measurements.

This study is very important due to the use of such hydrogels ( $\mathrm{pH}$ sensitive) in the medical field as devices for controlled-release drugs and also in the environmental protection and sustainable development field for wastewater purification.

Supplementary Materials: The following are available online at https: / www.mdpi.com/article / 10.3390/ma14092305/s1, Figure S1: The appearance of hy-drogels at different pH values: (a) SH sample (10\%MAA-2\%BIS-0\%MMT); (b) H1\%BIS sample (10\%MAA-1\%BIS-0\%MMT); (c) H2\%MMT (10\%MAA-2\%BIS-2\%MMT). Figure S2: Mechanism of the swelling process. Figure S3: SEM micrographs of lyophilized H2\%MMT samples swollen at different $\mathrm{pH}$ values: (a) $\mathrm{pH} 1.2$; (b) $\mathrm{pH}$ 5.4; (c) $\mathrm{pH} 7.4$.

Author Contributions: Conceptualization, C.M.N., M.T., R.I. and C.P.; Methodology, C.M.N. and M.T.; Validation, M.T. and C.P.; Formal Analysis, E.A., C.I.M. and S.P.; Investigation, C.M.N., C.S. and C.L.N.; Writing-Original Draft Preparation, C.M.N. and M.T.; Writing-Review and Editing, C.M.N., M.T., R.I. and C.P.; Supervision, M.T. and C.P. All authors have read and agreed to the published version of the manuscript.

Funding: This work was supported by the Romanian Ministry of Research and Innovation-MCI through the INCDCP ICECHIM Bucharest 2019-2022 Core Program PN. 19.23-Chem-Ergent, Project No. 19.23.02.01 (Advanced Polymeric Materials for Innovative Applications-MAT-INNOVA), and by a grant from the Ministry of Education and Research, CCCDI-UEFISCDI, project number PN-IIIP2-2.1-PED-2019-4216, within PNCDI III.

Institutional Review Board Statement: Not applicable.

Informed Consent Statement: Not applicable.

Data Availability Statement: Data sharing not available.

Acknowledgments: We thank Ana-Maria Albu and Bogdan Trica for their valuable support.

Conflicts of Interest: The authors declare no conflict of interest. 


\section{References}

1. Rizwan, M.; Yahya, R.; Hassan, A.; Yar, M.; Azzahari, A.; Selvanathan, V.; Sonsudin, F.; Abouloula, C. PH Sensitive Hydrogels in Drug Delivery: Brief History, Properties, Swelling, and Release Mechanism, Material Selection and Applications. Polymers 2017, 9, 137. [CrossRef]

2. de Lima, C.S.A.; Balogh, T.S.; Varca, J.P.R.O.; Varca, G.H.C.; Lugão, A.B.; Camacho-Cruz, L.A.; Bucio, E.; Kadlubowski, S.S. An Updated Review of Macro, Micro, and Nanostructured Hydrogels for Biomedical and Pharmaceutical Applications. Pharmaceutics 2020, 12, 970. [CrossRef]

3. Neethu, T.M.; Dubey, P.K.; Kaswala, A.R. Prospects and Applications of Hydrogel Technology in Agriculture. Int. J. Curr. Microbiol. Appl. Sci. 2018, 7, 3155-3162. [CrossRef]

4. Hao, G.-P.; Hippauf, F.; Oschatz, M.; Wisser, F.M.; Leifert, A.; Nickel, W.; Mohamed-Noriega, N.; Zheng, Z.; Kaskel, S. Stretchable and Semitransparent Conductive Hybrid Hydrogels for Flexible Supercapacitors. ACS Nano 2014, 8, 7138-7146. [CrossRef]

5. Quattrone, A.; Czajka, A.; Sibilla, S. Thermosensitive Hydrogel Mask Significantly Improves Skin Moisture and Skin Tone; Bilateral Clinical Trial. Cosmetics 2017, 4, 17. [CrossRef]

6. Zhao, L.Z.; Zhou, C.H.; Wang, J.; Tong, D.S.; Yu, W.H.; Wang, H. Recent Advances in Clay Mineral-Containing Nanocomposite Hydrogels. Soft Matter 2015, 11, 9229-9246. [CrossRef]

7. Panic, V.; Adnadjevic, B.; Velickovic, S.; Jovanovic, J. The Effects of the Synthesis Parameters on the Xerogels Structures and on the Swelling Parameters of the Poly(Methacrylic Acid) Hydrogels. Chem. Eng. J. 2010, 156, 206-214. [CrossRef]

8. García, D.M.; Escobar, J.L.; Bada, N.; Casquero, J.; Hernáez, E.; Katime, I. Synthesis and Characterization of Poly(Methacrylic Acid) Hydrogels for Metoclopramide Delivery. Eur. Polym. J. 2004, 40, 1637-1643. [CrossRef]

9. Panic, V.V.; Madzarevic, Z.P.; Volkov-Husovic, T.; Velickovic, S.J. Poly(Methacrylic Acid) Based Hydrogels as Sorbents for Removal of Cationic Dye Basic Yellow 28: Kinetics, Equilibrium Study and Image Analysis. Chem. Eng. J. 2013, 217, 192-204. [CrossRef]

10. Bashir, S.; Teo, Y.Y.; Ramesh, S.; Ramesh, K. Synthesis, Characterization, Properties of N-Succinyl Chitosan-g-Poly (Methacrylic Acid) Hydrogels and in Vitro Release of Theophylline. Polymer 2016, 92, 36-49. [CrossRef]

11. Güler, M.A.; Gök, M.K.; Figen, A.K.; Özgümüş, S. Swelling, Mechanical and Mucoadhesion Properties of Mt/Starch-g-PMAA Nanocomposite Hydrogels. Appl. Clay Sci. 2015, 112-113, 44-113. [CrossRef]

12. Qi, X.; Wei, W.; Li, J.; Liu, Y.; Hu, X.; Zhang, J.; Bi, L.; Dong, W. Fabrication and Characterization of a Novel Anticancer Drug Delivery System: Salecan/Poly(Methacrylic Acid) Semi-Interpenetrating Polymer Network Hydrogel. ACS Biomater. Sci. Eng. 2015, 1, 1287-1299. [CrossRef]

13. Adnadjevic, B.; Tasic, G.; Jovanovic, J. Kinetic of Non-Isothermal Dehydration of Equilibrium Swollen Poly(Acrylic Acid-CoMethacrylic Acid) Hydrogel. Thermochim. Acta 2011, 512, 157-162. [CrossRef]

14. Constantin, M.; Bucatariu, S.; Harabagiu, V.; Popescu, I.; Ascenzi, P.; Fundueanu, G. Poly(N-Isopropylacrylamide-Co-Methacrylic Acid) PH/Thermo-Responsive Porous Hydrogels as Self-Regulated Drug Delivery System. Eur. J. Pharm. Sci. 2014, 62, 86-95. [CrossRef] [PubMed]

15. Bajpai, S.K.; Singh, S. Analysis of Swelling Behavior of Poly(Methacrylamide-Co-Methacrylic Acid) Hydrogels and Effect of Synthesis Conditions on Water Uptake. React. Funct. Polym. 2006, 66, 431-440. [CrossRef]

16. Morishita, M.; Goto, T.; Peppas, N.A.; Joseph, J.I.; Torjman, M.C.; Munsick, C.; Nakamura, K.; Yamagata, T.; Takayama, K.; Lowman, A.M. Mucosal Insulin Delivery Systems Based on Complexation Polymer Hydrogels: Effect of Particle Size on Insulin Enteral Absorption. J. Control. Release 2004, 97, 115-124. [CrossRef] [PubMed]

17. Zhumagaliyeva, S.N.; Iminova, R.S.; Kairalapova, G.Z.; Beysebekov, M.M.; Beysebekov, M.K.; Abilov, Z.A. Composite PolymerClay Hydrogels Based on Bentonite Clay and Acrylates: Synthesis, Characterization and Swelling Capacity. Eurasian Chem. Technol. J. 2017, 19, 279-288. [CrossRef]

18. Junior, C.R.F.; de Moura, M.R.; Aouada, F.A. Synthesis and Characterization of Intercalated Nanocomposites Based on Poly(Methacrylic Acid) Hydrogel and Nanoclay Cloisite-Na ${ }^{+}$for Possible Application in Agriculture. J. Nanosci. Nanotechnol. 2017, 17, 5878-5883. [CrossRef]

19. Ianchis, R.; Ninciuleanu, C.; Gifu, I.; Alexandrescu, E.; Somoghi, R.; Gabor, A.; Preda, S.; Nistor, C.; Nitu, S.; Petcu, C.; et al. Novel Hydrogel-Advanced Modified Clay Nanocomposites as Possible Vehicles for Drug Delivery and Controlled Release. Nanomaterials 2017, 7, 443. [CrossRef]

20. Munteanu, T.; Ninciuleanu, C.M.; Gifu, I.C.; Trica, B.; Alexandrescu, E.; Gabor, A.R.; Preda, S.; Petcu, C.; Nistor, C.L.; Nitu, S.G.; et al. The Effect of Clay Type on the Physicochemical Properties of New Hydrogel Clay Nanocomposites. In Current Topics in the Utilization of Clay in Industrial and Medical Applications; Zoveidavianpoor, M., Ed.; InTech: London, UK, 2018; ISBN 978-1-78923-728-3.

21. Sen, M.; Yakar, A.; Güven, O. Determination of Average Molecular Weight between Cross-Links (Mc) from Swelling Behaviours of Diprotic Acid-Containing Hydrogels. Polymer 1999, 40, 2969-2974. [CrossRef]

22. Richbourg, N.R. The Swollen Polymer Network Hypothesis: Quantitative Models of Hydrogel Swelling, Stiffness, and Solute Transport. Prog. Polym. Sci. 2020, 105, 101243. [CrossRef]

23. Safronov, A.P.; Adamova, L.V.; Blokhina, A.S.; Kamalov, I.A.; Shabadrov, P.A. Flory-Huggins Parameters for Weakly Crosslinked Hydrogels of Poly(Acrylic Acid) and Poly(Methacrylic Acid) with Various Degrees of Ionization. Polym. Sci. Ser. A 2015, 57, 33-42. [CrossRef] 
24. Jerca, F.A.; Anghelache, A.M.; Ghibu, E.; Cecoltan, S.; Trusca, R.; Vasile, E.; Teodorescu, M.; Vuluga, D.M.; Jerca, V.V. Poly(2Isopropenyl-2-Oxazoline) Hydrogels for Biomedical Applications. Chem. Mater. 2018, 30, 7938-7949. [CrossRef]

25. Poly(Methacrylic Acid). Available online: http://polymerdatabase.com/polymers/polymethacrylicacid.html (accessed on 3 February 2021).

26. Orakdogen, N.; Okay, O. Effect of Initial Monomer Concentration on the Equilibrium Swelling and Elasticity of Hydrogels. Eur. Polym. J. 2006, 42, 955-960. [CrossRef]

27. Mezger, T.G. The Rheology Handbook: For Users of Rotational and Oscillatory Rheometers; Vincentz Network GmbH \& Co KG: Hannover, Germany, 2006; ISBN 978-3-87870-174-3.

28. Mithra, K.; Khandai, S.; Mishra, B.; Jena, S.S. Effect of Polymer Concentration on Structure and Rheology of Poly (Sodium Acrylate) Hydrogels. In AIP Conference Proceedings 1942; American Institute of Physics: Mumbai, India, 2018 ; p. 040009.

29. Teodorescu, M.; Lungu, A.; Stanescu, P.O. Preparation and Properties of Novel Slow-Release NPK Agrochemical Formulations Based on Poly(Acrylic Acid) Hydrogels and Liquid Fertilizers. Ind. Eng. Chem. Res. 2009, 48, 6527-6534. [CrossRef]

30. Salimi-Kenari, H.; Mollaie, F.; Dashtimoghadam, E.; Imani, M.; Nyström, B. Effects of Chain Length of the Cross-Linking Agent on Rheological and Swelling Characteristics of Dextran Hydrogels. Carbohydr. Polym. 2018, 181, 141-149. [CrossRef]

31. Yu, C.; Tang, X.; Liu, S.; Yang, Y.; Shen, X.; Gao, C. Laponite Crosslinked Starch/Polyvinyl Alcohol Hydrogels by Freezing/Thawing Process and Studying Their Cadmium Ion Absorption. Int. J. Biol. Macromol. 2018, 117, 1-6. [CrossRef]

32. Bajpai, S.; Chand, N.; Mahendra, M. The Adsorptive Removal of a Cationic Drug from Aqueous Solution Using Poly (Methacrylic Acid) Hydrogels. Water SA 2014, 40, 49-56. [CrossRef]

33. Ltek, A.G.; Ki, T.S. Poly(methacrylic) Acid and $\gamma$-methacryloxypropyltrimethoxy Silane/Clay Nanocomposites Prepared by In-Situ Polymerization. Turk. J. Chem. 2002, 26, 925-937.

34. Reguieg, F.; Ricci, L.; Bouyacoub, N.; Belbachir, M.; Bertoldo, M. Thermal Characterization by DSC and TGA Analyses of PVA Hydrogels with Organic and Sodium MMT. Polym. Bull. 2020, 77, 929-948. [CrossRef]

35. Pourjavadi, A.; Samadi, M.; Ghasemzadeh, H. Fast-Swelling Superabsorbent Hydrogels from Poly(2-Hydroxy Ethyl Acrylate-CoSodium Acrylate) Grafted on Starch. Starch Stärke 2008, 60, 79-86. [CrossRef] 